\title{
Reduced CXCL4/PF4 expression as a driver of increased human hematopoietic stem and progenitor cell proliferation in polycythemia vera
}

\author{
Fabienne Meier-Abt $\mathbb{0}^{1,2}$, Witold E. Wolski ${ }^{3,4}$, Ge Tan $\mathbb{0}^{3}$, Sandra Kummer ${ }^{1}$, Sabine Amon², Markus G. Manz ${ }^{1}$,
} Ruedi Aebersold ${ }^{2,5}$ and Alexandre P. A. Theocharides ${ }^{1}$

Dear Editor,

Polycythemia vera (PV) is a myeloproliferative neoplasm (MPN) marked by hyperproliferation of all myeloid cell lineages and characterized by activated JAKSTAT signaling due to an activating mutation in $J A K 2^{1}$. Disease-driving pathogenic changes in MPNs are thought to arise in hematopoietic stem cells (HSCs) that give rise to the diseased clonal progeny ${ }^{2,3}$. We recently developed a new data-independent acquisition (DIA) mass spectrometry (MS) technology for rare human hematopoietic stem and progenitor cell (HSPC) subpopulations $^{4}$. This DIA-MS proteomic analysis was applied to human hematopoietic stem/multipotent progenitor cells (HSC/MPPs) and common myeloid/ megakaryocyte-erythrocyte progenitors (CMP/MEPs) isolated from 123 blood samples of $18 \mathrm{PV}$ patients and 21 controls (Supplementary Table 1). The proteomic dataset was complemented with RNA-sequencing data of the same patient and control samples.

Comparing the proteomic and transcriptomic datasets in the corresponding patient and control samples demonstrated mainly positive correlations: $70 \%$ of genes had positive Spearman's correlation coefficients for protein and RNA expression. In line with our previous observations in HSC/MPPs of healthy stem cell donors ${ }^{4}$, $30 \%$ of genes with altered expression in PV patients and their controls demonstrated negative correlation

\footnotetext{
Correspondence: Fabienne Meier-Abt (fabienne.meier-abt@usz.ch)

${ }^{1}$ Department of Medical Oncology and Hematology, University Hospital Zurich and University of Zurich, Zurich, Switzerland

${ }^{2}$ Department of Biology, Institute of Molecular Systems Biology, ETH Zurich, Zurich, Switzerland

Full list of author information is available at the end of the article

These authors contributed equally: Markus G. Manz, Ruedi Aebersold,

Alexandre P. A. Theocharides
}

coefficients for protein and RNA expression, indicating additional information gained by proteomic compared to transcriptomic data.

\section{Downregulation of megakaryocyte differentiation and upregulation of cell proliferation in PV HSPCs and its reversal upon treatment with hydroxyurea (HU)}

To further examine the added information provided by protein compared to RNA expression data, we performed enrichment analyses for gene ontologies in HSPC subpopulations of PV patients on protein and RNA levels. Megakaryocyte differentiation and regulation was significantly downregulated in untreated PV patients at the protein level, but not at the RNA level in all HSPCs analyzed (Fig. 1A with individual gene ontology protein members shown in Fig. 1B). Similarly, RNAs and proteins implicated in DNA replication and G1/S transition of the mitotic cell cycle were discordantly regulated in HSC/MPPs of untreated PV patients (Fig. 1A). In contrast to the discordant protein and RNA expression in these gene sets, we observed concordant expression on the protein and RNA level for erythrocyte differentiation, regulation, development and maturation, receptor signaling via JAK-STAT, interferon-gamma signaling, cholesterol biosynthetic process, and TGF $\beta$ and MAPK signaling and regulation in HSPCs of untreated PV patients compared to controls (Fig. 1A).

Cytoreductive therapy with hydroxyurea reverted downregulation of megakaryocyte differentiation and regulation on protein level (Fig. 1A, B). It also reversed upregulation of cell proliferation (DNA replication, G1/S transition of the mitotic cell cycle, DNA repair) on RNA 
(A)

\begin{tabular}{|c|c|c|c|c|c|c|c|c|}
\hline & & & & & & & & \\
\hline & \multicolumn{8}{|c|}{ NES } \\
\hline & \multicolumn{2}{|c|}{$\begin{array}{l}\text { PV.UT.HSC/MPP- } \\
\text { Control.HSC/MPP }\end{array}$} & \multicolumn{2}{|c|}{$\begin{array}{l}\text { PV.UT.CMP/MEP- } \\
\text { Control.CMP/MEP }\end{array}$} & \multicolumn{2}{|c|}{$\begin{array}{l}\text { PV.HU.HSC/MPP- } \\
\text { PV.UT.HSC/MPP }\end{array}$} & \multicolumn{2}{|c|}{$\begin{array}{l}\text { PV.HU.CMP/MEP- } \\
\text { PV.UT.CMP/MEP }\end{array}$} \\
\hline & RNA & Prt & RNA & Prt & RNA & Prt & RNA & Prt \\
\hline Erythrocyte development \& maturation & 0.65 & 1.38 & 1.32 & 1.27 & 1.20 & 1.05 & 0.73 & -1.45 \\
\hline Erythrocyte differentiation \& regulation & 0.77 & 0.77 & 0.72 & 1.10 & 1.10 & -1.31 & 1.37 & -1.85 \\
\hline HSC differentiation \& regulation & 0.65 & 0.88 & 1.10 & 0.61 & -1.30 & -1.33 & 0.81 & -1.29 \\
\hline MEGA differentiation \& regulation & 0.67 & -1.90 & 0.95 & -1.86 & 1.02 & 1.55 & 1.12 & 1.50 \\
\hline DNA replication & 2.16 & -1.31 & 2.24 & 0.64 & -2.28 & -2.20 & -1.52 & -0.60 \\
\hline G1/S transition of mitotic cell cycle & 1.97 & -1.21 & 2.21 & -0.80 & -2.15 & -1.77 & -1.31 & 0.55 \\
\hline DNA repair & 1.61 & 0.63 & 1.72 & 0.89 & -1.66 & -1.44 & -1.09 & 0.58 \\
\hline Receptor signaling pathway via JAK-STAT & 0.89 & 1.75 & 1.13 & 1.36 & 1.00 & -0.86 & 0.37 & -0.86 \\
\hline Interferon-gamma-mediated signaling pathway & 1.32 & 1.59 & 1.69 & 1.99 & 1.67 & 1.57 & 1.80 & 1.28 \\
\hline TGFbeta signaling pathway \& regulation & -1.40 & -1.34 & -1.69 & -1.68 & 1.46 & 0.88 & 1.30 & 1.74 \\
\hline BMP signaling pathway \& regulation & -0.87 & 0.72 & -1.71 & 0.79 & 1.55 & -1.62 & 1.51 & -1.05 \\
\hline MAPK activity \& regulation & -1.34 & -0.99 & -1.72 & -0.77 & 1.43 & -0.81 & 1.42 & 0.65 \\
\hline PI3K signaling \& regulation & -1.00 & 0.78 & -1.11 & -1.06 & 1.57 & -0.85 & 1.48 & -0.96 \\
\hline Cholesterol biosynthetic process & 1.40 & 1.38 & 1.48 & 1.69 & -1.95 & 1.05 & -1.73 & 0.74 \\
\hline
\end{tabular}

(B)

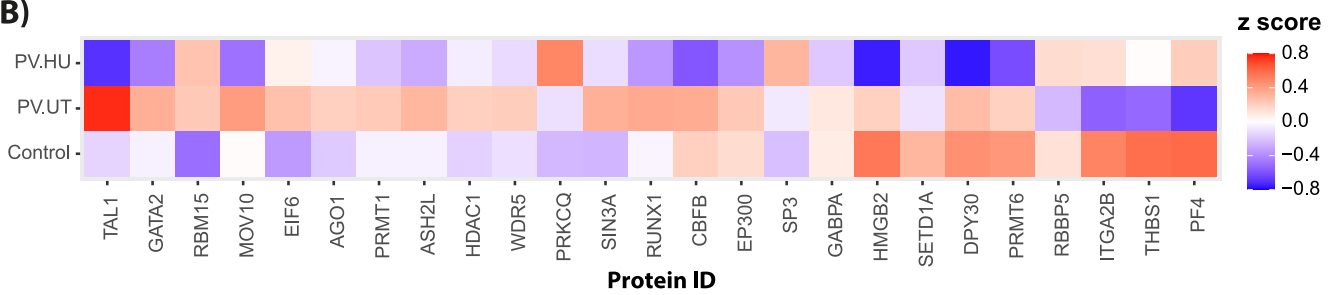

(C)

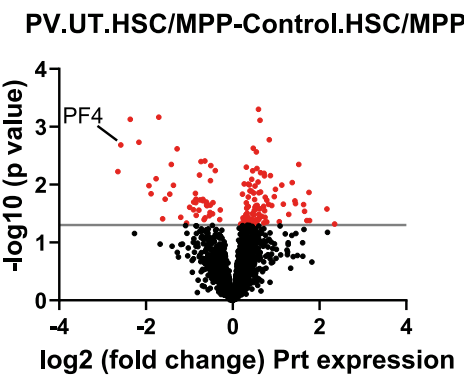

(F)
(D)

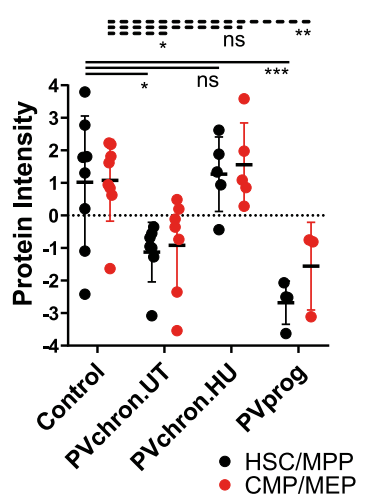

(E)

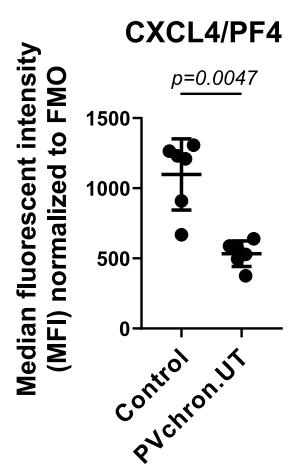

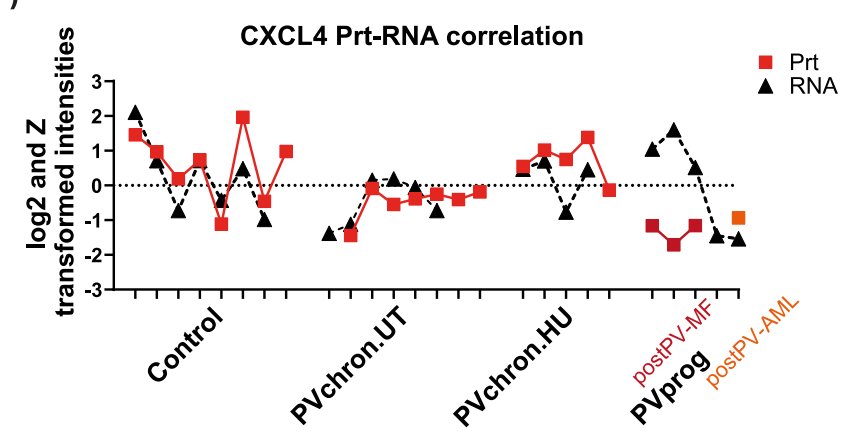

Fig. 1 (See legend on next page.)

level and downregulation of TGF $\beta$ signaling on protein and RNA level (Fig. 1A). Erythrocyte differentiation and regulation were reversed by hydroxyurea on the protein but not RNA level, whereas interferon-gamma signaling was not affected by the treatment of patients with hydroxyurea (Fig. 1A). 
(see figure on previous page)

Fig. 1 Comparative unbiased proteomic analysis identifies downregulation of CXCL4/PF4 in HSC/MPPs of PV patients. A Gene set enrichment analysis (GSEA) comparing untreated PV patients against controls (PV.UT.HSC/MPP versus Control.HSC/MPP; PV.UT.CMP/MEP versus Control.CMP/MEP) and assessing for the effect of treatment with hydroxyurea (PV.HU.HSC/MPP versus PV.UT.HSC/MPP; PV.HU.CMP/MEP versus PV.UT.CMP/MEP). Shown are normalized enrichment scores (NES) for individual gene sets. Significantly upregulated gene sets are marked in red color, significantly downregulated gene sets are marked in blue color. Only RNAs and proteins expressed in at least half of the replicates in both comparison groups were considered. UT untreated, HU patient under treatment with hydroxyurea. B Heatmap of proteins enriched in different patient and control groups for megakaryocyte differentiation and regulation. C Volcano plot of protein intensity fold changes and p-values comparing HSC/MPPs of untreated PV patients (PV.UT.HSC/MPP) against controls (Control.HSC/MPP). D Normalized protein intensities for CXCL4/ PF4 in the subgroups of controls, chronic PV patients without cytoreductive therapy (PVchron.UT), chronic PV patients with hydroxyurea therapy (PVchron.HU), and progressed PV patients (PVprog). Error bars represent standard deviations. ${ }^{*}$ adj. $P<0.05$; ${ }^{* *}$ adj. $P<0.01 ;{ }^{* * *}$ adj. $P<0.001$ Peptide profiles for CXCL4/PF4 are provided in Supplementary Fig. 1. E Graphical summary of intracellular FACS staining experiments for CXCL4/ PF4 in six untreated chronic PV patients and age- and gender-matched controls. Error bars represent standard deviations. F Normalized CXCL4/ PF4 expression values for protein and RNA in individual patient and control samples. Discrepant high RNA and low protein levels were seen in progressed PV patients with fibrosis (post-PV MF) but not in post-PV AML.

\section{Reduced CXCL4/PF4 expression in HSC/MPPs of untreated PV patients}

To better characterize the molecular phenotype underlying PV stem and progenitor cell biology, we next focused on individual differentially regulated proteins in HSC/MPPs of untreated PV patients compared to controls. Consistent with the downregulation of megakaryocyte differentiation and regulation observed in the gene ontology enrichment analysis (Fig. 1A, B), we observed significant downregulation of the megakaryocytic lineage protein CXCL4/PF4 (platelet factor 4) (Fig. 1C, D and Supplementary Fig. 1). Intracellular flow cytometry confirmed the downregulation of CXCL4/PF4 in HSC/MPPs of untreated chronic phase PV patients in comparison to age- and gender-matched controls (Fig. 1E). Treatment with hydroxyurea abrogated the downregulation of CXCL4/PF4 protein expression in PV HSC/MPPs (Fig. 1D).

Since all PV patients analyzed in this study carried the JAK2V617F mutation, we tested for a potential direct relationship between the JAK2V617F allele burden and CXCL4/PF4 protein expression. No significant correlation was observed (Supplementary Fig. 2), suggesting that reduced CXCL4/PF4 expression in $\mathrm{PV}$ is a JAK2V617F allele burden-independent disease manifestation.

CXCL4/PF4 did not show equally significant expression changes on the RNA level as on the protein level. Exploring protein-RNA-correlations in more detail, we observed that protein and RNA levels became discordant upon disease progression (Fig. 1F). Upregulated RNA and downregulated protein levels for CXCL4/PF4 were observed in HSC/MPPs of post-PV myelofibrosis (MF) patients but not in post-PV acute myeloid leukemia (AML) stem cells. In controls and non-progressed PV patients, protein and RNA levels of CXCL4/PF4 were better aligned even if not systematically coordinated (Fig. 1F).
Decreased ELF1 and USF2 activity explains reduced CXCL4/ PF4 expression, which is linked to downregulated TGF $\beta$ signaling and loss of stem cell quiescence

We next assessed the activities of transcription factors (TFs), which bind the CXCL4/PF4 promoter and may regulate CXCL4/PF4 expression in PV HSC/MPPs (Fig. 2A $)^{5-8}$. We found a significantly decreased activity for ELF1 and to a lesser extent also for USF2 in HSC/ MPPs of untreated PV compared to controls and HUtreated PV (Fig. 2A), suggesting a potential link between diminished ELF1 and USF2 activity and a reduction of CXCL4/PF4 expression in HSC/MPPs of untreated PV patients $^{5,8}$. Upon progression to post-PV MF, the activities of the TFs USF2 and also NKX2-2 increased, potentially explaining upregulated RNA levels of CXCL4/ PF4 in these patients (Supplementary Fig. 3$)^{5}$.

Reduced CXCL4/PF4 expression in HSC/MPPs of PV patients was associated with significant upregulation of genes downregulated in stem cell quiescence both on RNA (Fig. 2B, D) and protein (Supplementary Fig. 4A and 4C) level ${ }^{9}$. This pattern was reversed by treatment of patients with cytoreductive hydroxyurea (Fig. 2C, D and Supplementary Fig. 4B, C). Mechanistically, CXCL4/PF4 upregulates TGF $\beta^{10}$, which promotes stem cell quiescence $^{11}$. We observed downregulation of TGF $\beta$ signaling in untreated PV patients that also exhibited downregulated CXCL4/PF4 levels (Fig. 1A). These data suggest that reduced CXCL4/PF4 expression in PV HSC/MPPs leads to the cessation of stem cell quiescence via downregulation of TGF $\beta$ signaling.

\section{CXCL4/PF4 inhibits colony formation of HSC/MPPs isolated from untreated chronic phase PV patients}

To assess the functional relevance of CXCL4/PF4 downregulation and to examine its causal link to $\mathrm{HSC} /$ MPP proliferation and differentiation in PV, we cultured HSC/MPPs of untreated chronic phase PV patients in the presence or absence of CXCL4/PF4 using two independent 
(A)

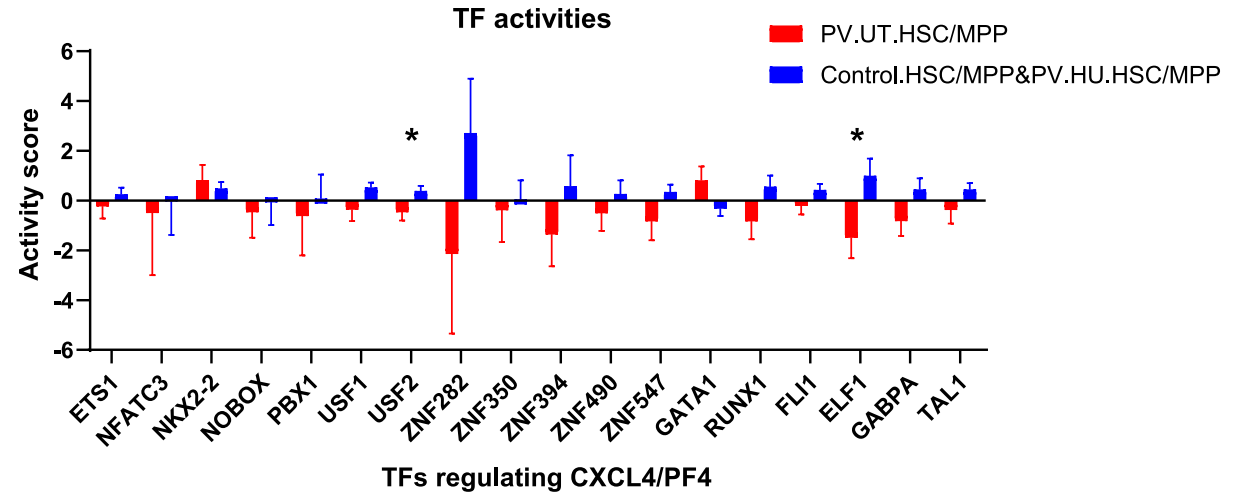

(B)

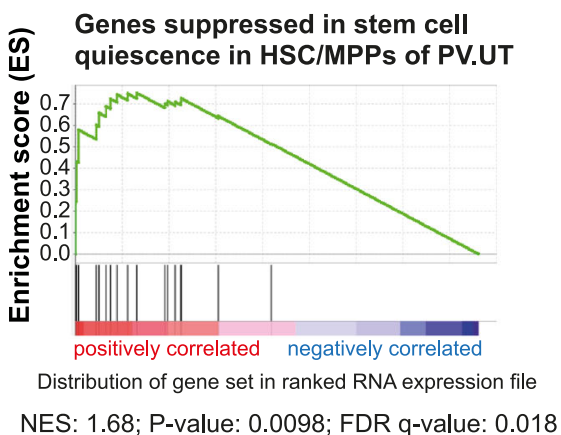

(C)

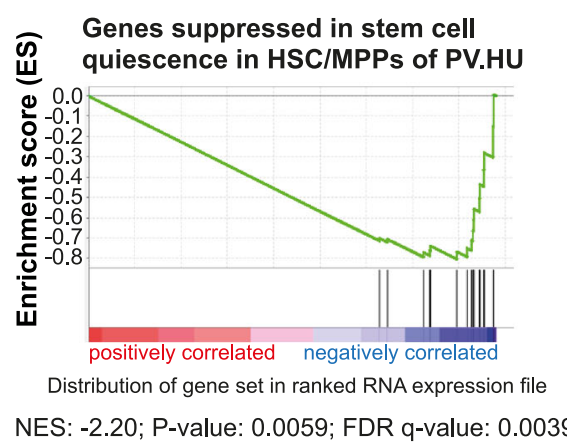

(D)

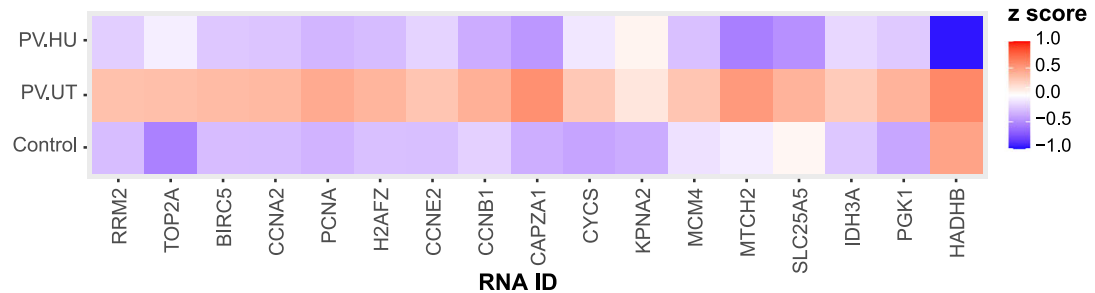

(E)

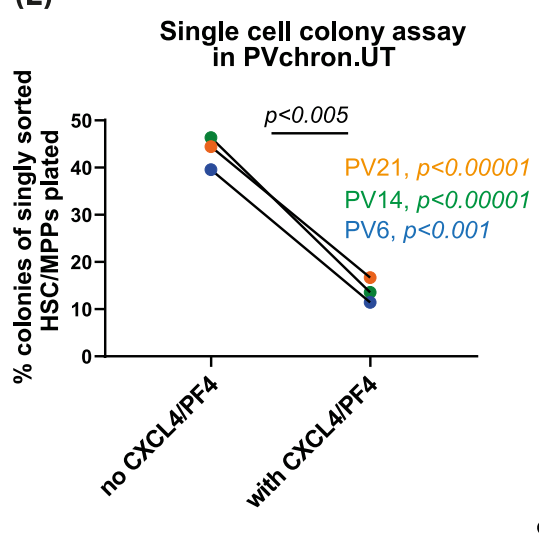

(F)
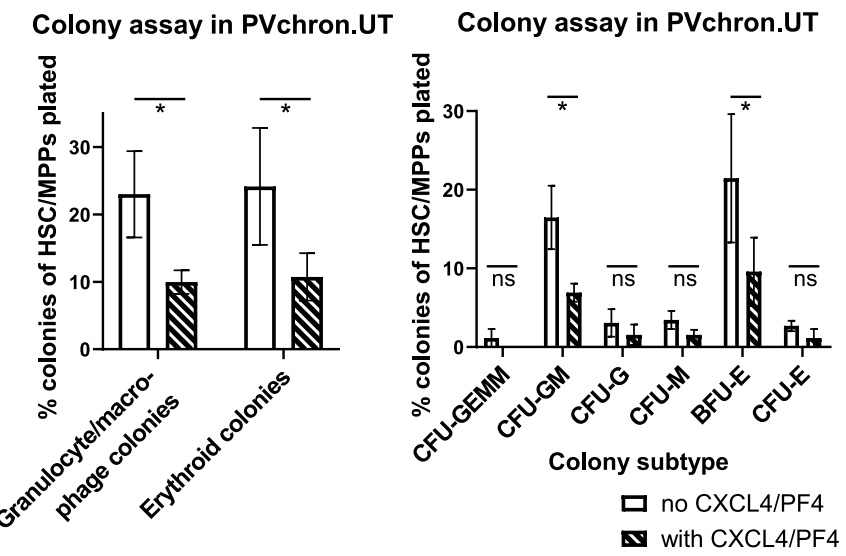

Fig. 2 (See legend on next page.)

colony formation assays. Supplementation with CXCL4/ PF4 inhibited colony formation of singly sorted PV HSC/ MPPs after 7 days in the cytokine-enriched serum-free medium by $63-71 \%$ (Fig. 2E). In methylcellulose assays, a $54-61 \%$ inhibition of total colony formation was observed upon reconstitution/treatment with CXCL4/PF4 
(see figure on previous page)

Fig. 2 CXCL4/PF4 protein is associated with transcriptional stem cell quiescence in untreated PV and inhibits the colony-formation capacity of PV HSC/MPPs. A Activity analysis of transcription factors (TFs) regulating CXCL4/PF4 expression showed significantly downregulated activity for the TF ELF1 and to a lesser degree for the TF USF2 in untreated PV patients compared to controls and HU-treated PV patients. Error bars represent standard errors. ${ }^{*} P<0.05$. B Gene set enrichment analysis showed strong and significant enrichment for genes downregulated in stem cell quiescence in HSC/MPPs of untreated PV patients (PV.UT) compared to HSC/MPPs of controls, suggesting cessation of stem cell quiescence in PV stem cells. C Treatment of patients with hydroxyurea (PV.HU.HSC/MPP versus PV.UT.HSC/MPP) reversed the loss of stem cell quiescence in the PV HSC/MPP subpopulation. NES normalized enrichment score. D Heatmap of RNAs enriched in different patient/control groups for genes downregulated in stem cell quiescence. The corresponding enrichment plots and heatmap for the protein level are shown in Supplementary Fig. 4AC. E Functional single-cell CXCL4/PF4 assay: colony growth of FACS-sorted HSC/MPPs from untreated chronic PV patients (PVchron.UT) singly incubated in cytokine-enriched serum-free medium was assessed in presence or absence of CXCL4/PF4. Colony growth as depicted was evaluated in 864 wells after 7 days of incubation. In individual patients, significance was determined using Fisher's exact test; significance overall was calculated using two-tailed and paired Student's $t$ test (see Supplementary Methods). A strong growth inhibitory role of CXCL4/PF4 was observed.

F Methylcellulose colony assay: the growth of different myeloid colony subtypes from FACS-sorted HSC/MPPs of untreated chronic PV patients (PVchron.UT) was evaluated in presence or absence of CXCL4/PF4. Reductions in colony growth due to CXCL4/PF4 protein were observed for all colony subtypes with significant inhibitions seen in the CFU-GM and BFU-E colony subtypes as well as in composite granulocyte/macrophage (CFUGM and CFU-G and CFU-M) and erythroid (BFU-E and CFU-E) colonies. Relative numbers of colonies related to the total cell numbers plated are plotted. Error bars represent standard deviations. ${ }^{*} P<0.05$. CFU-GEMM (mixed colonies), CFU-GM colony-forming unit-granulocyte/macrophage, CFU-G colony-forming unit-granulocyte, CFU-M colony-forming unit-macrophage, BFU-E burst-forming unit-erythroid, CFU-E colony-forming unit-erythroid.

for FACS-sorted HSC/MPPs from PV patients (Fig. 2F). Detailed analyses for colony subtypes as defined by Manz et al. ${ }^{12}$ showed significant reductions in colony growth from PV HSC/MPPs upon exposure to supplemental CXCL4/PF4 for CFU-GM (colony-forming unit-granulocyte/macrophage) and BFU-E (burst-forming unit-erythroid) (Fig. 2F). These results demonstrate that CXCL4/PF4 is linked to HSC/MPP proliferation and differentiation in PV, and reduced CXCL4/PF4 expression in HSC/MPPs may contribute to the proliferative state of PV.

\section{Discussion}

This study identifies reduced CXCL4/PF4 protein expression in HSC/MPPs of untreated PV patients compared to controls. Treatment of patients with cytoreductive hydroxyurea abrogated this effect. We demonstrated transcriptional cessation of stem cell quiescence to be associated with reduced CXCL4/PF4 expression in PV HSC/MPPs, and supplementation with CXCL4/ PF4 strongly inhibited the in vitro colony-formation capacity of HSC/MPPs from PV patients. These findings extend previous reports in healthy hematopoietic stem cells and non-PV JAK2-mutated erythroblast-like cells ${ }^{13}$. CXCL4/PF4-/- mice were shown to exhibit increased numbers and proliferation of HSCs and MPPs ${ }^{14}$, and high CXCL4/PF4 levels were found to strongly inhibit hematopoiesis in non-PV mice ${ }^{14}$. The inhibitory role of CXCL4/ PF4 on HSPC proliferation in our PV patients supports a key role of downregulated CXCL4/PF4 for the proliferative phenotype of PV HSC/MPPs.

Mechanistically, we observed downregulation of TGF $\beta$ signaling in untreated PV patients showing reduced CXCL4/PF4 expression. CXCL4/PF4 was previously shown to activate stem cell quiescence-inducing TGF $\beta^{10,11}$. Upstream of CXCL4/PF4, we identified reduced activity of the CXCL4/PF4-regulating transcription factors $E L F 1^{8}$ and $\mathrm{USF}^{5}$ in HSC/MPPs of untreated PV patients. Our data thus support a model in which decreased ELF1 and USF2 activity in PV HSC/MPPs leads to reduced CXCL4/PF4 expression, which leads to decreased TGF $\beta$ signaling and concomitant cessation of stem cell quiescence. Upon progression to post-PV MF, USF2 activity increased, explaining elevated CXCL4/PF4 RNA levels in this patient group.

The PV disease stages and controls were better separated by CXCL4/PF4 protein expression than by CXCL4/ PF4 RNA expression. Whereas transcriptomics reinforced the proteomic results for CXCL4/PF4 in chronic phase $\mathrm{PV}$, discrepant RNA and protein levels were observed in HSC/MPPs of post-PV MF patients. Our findings in fibrotic patients are in line with recent observations of upregulated CXCL4/PF4 RNA levels in co-cultured HSPCs of a murine PMF model ${ }^{15}$. The congruent CXCL4/PF4 protein and RNA expression in control, chronic PV, and post-PV AML, but not in post-PV MF suggests post-transcriptional regulation at the base of discrepant RNA and protein levels in post-PV MF.

In summary, this study identified downregulation of CXCL4/PF4 expression in HSC/MPPs with the cessation of stem cell quiescence through downregulation of TGF $\beta$ signaling as a potential new driver of the proliferative state of PV.

\section{Acknowledgements}

We'd like to acknowledge Tatjana Sajic, Junyan Lu, and Thorsten Zenz for bioinformatic advice and Thijs Wildschut for critical reading of the manuscript. Furthermore, we'd like to thank Patrizia Belleda and Asuka Fry for help with patient sample processing. We would also like to thank the FACS facilities of the ETHZ and the University of Zurich for their assistance. This research was funded by grants to F.M.-A. from PHRT (ETHZ), Filling-the-Gap (UZH),

Promedica and Krebsliga Zurich. Work was also supported by the Swiss

National Science Foundation (grant no. 3100A0-688 107679) to R.A.. A.P.A.T. is supported by the Professor Dr. Max Cloëtta Foundation. 


\section{Author details}

${ }^{1}$ Department of Medical Oncology and Hematology, University Hospital Zurich and University of Zurich, Zurich, Switzerland. 'Department of Biology, Institute of Molecular Systems Biology, ETH Zurich, Zurich, Switzerland. ${ }^{3}$ Functional Genomics Center Zurich, University and ETH Zurich, Zurich, Switzerland. ${ }^{4}$ Swiss Institute of Bioinformatics, Lausanne, Switzerland. ${ }^{5}$ Faculty of Science, University of Zurich, Zurich, Switzerland

\section{Author contributions}

F.M.-A., M.G.M., R.A., and A.P.A.T. conceived of the study. F.M.-A., S.K., and S.A. performed experiments. F.M.-A., W.W., and G.T. analyzed the data. F.M.-A., M.G.M., R.A., and A.P.A.T. wrote the paper.

\section{Data availability}

Proteomic and transcriptomic data can be made available upon request to the corresponding author.

\section{Conflict of interest}

R.A. keeps shares of Biognosys AG which operates in the proteomics field. The remaining authors declare no competing interests.

\section{Publisher's note}

Springer Nature remains neutral with regard to jurisdictional claims in published maps and institutional affiliations.

Supplementary information The online version contains supplementary material available at https://doi.org/10.1038/s41408-021-00423-5.

Received: 13 November 2020 Revised: 5 January 2021 Accepted: 20 January 2021

Published online: 11 February 2021

\section{References}

1. Tefferi, A. \& Barbui, T. Polycythemia vera and essential thrombocythemia: 2019 update on diagnosis, risk-stratification and management. Am. J. Hematol. 94, 133-143 (2019).
2. Lundberg, P. et al. Myeloproliferative neoplasms can be initiated from a single hematopoietic stem cell expressing JAK2-V617F. J. Exp. Med. 211, 2213-2230 (2014).

3. Mead, A. J. \& Mullally, A. Myeloproliferative neoplasm stem cells. Blood 129 1607-1616 (2017)

4. Amon, S. et al. Sensitive quantitative proteomics of human hematopoietic stem and progenitor cells by data-independent acquisition mass spectrometry. Mol. Cell Proteomics. 18, 1454-1467 (2019).

5. Garcia-Alonso, L., Holland, C. H., Ibrahim, M. M., Turei, D. \& SaezRodriguez, J. Benchmark and integration of resources for the estimation of human transcription factor activities. Genome Res. 29, 1363-1375 (2019).

6. Vyas, P., Ault, K., Jackson, C. W., Orkin, S. H. \& Shivdasani, R. A. Consequences of GATA-1 deficiency in megakaryocytes and platelets. Blood 93, 2867-2875 (1999).

7. Aneja, K., Jalagadugula, G., Mao, G., Singh, A. \& Rao, A. K. Mechanism of platelet factor 4 (PF4) deficiency with RUNX1 haplodeficiency: RUNX1 is a transcriptional regulator of PF4. J. Thromb. Haemost. 9, 383-PF91 (2011).

8. Okada, Y. et al. Multiple ETS family proteins regulate PF4 gene expression by binding to the same ETS binding site. PLOS ONE 6, e24837 (2011).

9. Cheung, T. H. \& Rando, T. A. Molecular regulation of stem cell quiescence. Nat. Rev. Mol. Cell Biol. 14, 329-340 (2013).

10. Deng, S. et al. Non-platelet-derived CXCL4 differentially regulates cytotoxic and regulatory $T$ cells through $C X C R 3$ to suppress the immune response to colon cancer. Cancer Lett. 443, 1-12 (2019).

11. Blank, U. \& Karlsson, S. TGF-beta signaling in the control of hematopoietic stem cells. Blood 125, 3542-3550 (2015).

12. Manz, M. G., Miyamoto, T., Akashi, K. \& Weissman, I. L. Prospective isolation of human clonogenic common myeloid progenitors. Proc. Natl Acad. Sci. USA 99 11872-11877 (2002).

13. Han, Z. C. et al. Inhibitory effect of platelet factor 4 (PF4) on the growth of human erythroleukemia cells: proposed mechanism of action of PF4. J. Lab. Clin. Med. 120, 645-660 (1992).

14. Bruns, I. et al. Megakaryocytes regulate hematopoietic stem cell quiescence through CXCL4 secretion. Nat. Med. 20, 1315-1320 (2014).

15. Gleitz, $H$. et al. Increased CXCL4 expression in hematopoietic cells links inflammation and progression of bone marrow fibrosis in MPN. Blood 136, 2051-2064 (2020) 